\title{
Economic evaluation of posaconazole versus fluconazole prophylaxis in patients with graft-versus-host disease (GVHD) in the Netherlands
}

\author{
Jeroen P. Jansen • Amy K. O'Sullivan • \\ Elly Lugtenburg • Lambert F. R. Span • \\ Jeroen J. W. M. Janssen • Wiro B. Stam
}

Received: 3 August 2009 / Accepted: 4 March 2010/Published online: 10 April 2010

(C) The Author(s) 2010. This article is published with open access at Springerlink.com

\begin{abstract}
The objective of this study was to evaluate the cost-effectiveness of posaconazole versus fluconazole for the prevention of invasive fungal infections (IFI) in graftversus-host disease (GVHD) patients in the Netherlands. A decision analytic model was developed based on a doubleblind randomized trial that compared posaconazole with fluconazole antifungal prophylaxis in recipients of allogeneic HSCT with GVHD who were receiving immunosuppressive therapy (Ullmann et al., N Engl J Med 356:335-347, 2007). Clinical events were modeled with chance nodes
\end{abstract}

Financial Support This study was funded by Schering Plough Corporation.

J. P. Jansen $(\bowtie)$

Mapi Values,

133 Portland Street,

Boston, MA 02114, USA

e-mail: Jeroen.jansen@mapivalues.com

A. K. O'Sullivan

i3 Innovus,

Medford, MA, USA

E. Lugtenburg

Department of Hematology, Erasmus MC,

Rotterdam, The Netherlands

L. F. R. Span

Department of Hematology, University Medical Center,

Utrecht, The Netherlands

J. J. W. M. Janssen

VU University Medical Center,

Amsterdam, The Netherlands

W. B. Stam

Mapi Values,

Houten, The Netherlands reflecting probabilities of IFIs, IFI-related death, and death from other causes. Data on life expectancy, quality-of-life, medical resource consumption, and costs were obtained from the literature. The total cost with posaconazole amounted to $€ 9,428$ ( $95 \%$ uncertainty interval $€ 7,743-$ $11,388)$, which is $€ 4,566(€ 2,460-6,854)$ more than those with fluconazole. Posaconazole prophylaxis resulted in 0.17 $(0.02-0.36)$ quality adjusted life year (QALY) gained compared to fluconazole prophylaxis, corresponding to an incremental cost effectiveness ratio (ICER) of $€ 26,225$ per QALY gained. A scenario analysis demonstrated that at an increased background IFI risk (from 9\% to $15 \%$ ) the ICER was $€ 13,462$ per QALY. Given the underlying data and assumptions, posaconazole prophylaxis is expected to be cost-effective relative to fluconazole in recipients of allogeneic HSCT developing GVHD in the Netherlands. The cost-effectiveness of posaconazole depends on the IFI risk, which can vary by hospital.

Keywords Posaconazole $\cdot$ Cost-effectiveness Graft-versus-host disease $\cdot$ Prophylaxis

\section{Introduction}

Over the past two decades, the incidence of invasive fungal infections (IFI) has been rising [1]. The main risk groups for IFIs are patients experiencing prolonged, profound neutropenia following intensive chemotherapy for hematological malignancies and patients undergoing allogeneic haematopoietic stem cell transplantation (HSCT) [1]. Graft failure and graft-versus-host disease (GVHD), as well as the immunosuppression required for GVHD management, can exacerbate susceptibility to IFIs in HSCT patients [1]. 
Studies have reported invasive fungal infections as the primary cause of mortality associated with GVHD [2, 3]. Early diagnosis of fungal infections is difficult as nonspecific symptoms (fever) are often the only sign. Furthermore, antifungal treatment of established IFIs has a high failure rate $(60-70 \%)$ for allogeneic HSCT recipients $[3,4]$ and is very costly [5]. Hence, prophylaxis of IFIs is often considered as a valuable treatment strategy for neutropenic patients.

In the Netherlands, fluconazole is the preferred orally administered antifungal agent for prophylaxis of IFI. Prophylaxis with fluconazole reduces morbidity and mortality among recipients of allogeneic $\operatorname{HSCT}[6,7]$. One of the limitations of fluconazole is the lack of efficacy against aspergillus species, which together with Candida species are the most common causes of IFIs [8-10]. Posaconazole is a new-generation triazole which displays a broad spectrum of activity [3]. In two randomized controlled trials (RCT), the prophylactic efficacy relative to standard azoles was established in recipients of allogeneic HSCT [11] as well as in neutropenic patients undergoing remission-induction chemotherapy for acute myeloid leukemia (AML) and high-risk myelodysplastic syndrome (MDS) [12]. Based on these RCTs, posaconazole is licensed for prophylaxis of IFIs in these patient populations.

Since controlling the healthcare budget is a major priority for all healthcare systems, decisions related to reimbursement or budget allocation will be based not only on efficacy and safety of the available antifungal agents but also on the associated costs. Though the acquisition costs for posaconazole are higher relative to fluconazole, information on other medical resource consumption related to the treatment of fungal infections is required for a complete pharmacoeconomic picture. RCTs are generally not designed to provide this information. A model-based health economic study on the other hand is a well-accepted method to assess the cost-effectiveness of interventions. It allows for the integration of efficacy and safety data from clinical trials and medical resource consumption from other data sources and accounts for the uncertainties that result from such a combination of information. A recent economic evaluation demonstrated that posaconazole prophylaxis is expected to be cost-effective compared to standard azole in neutropenic AML/MDS patients in the Netherlands [13].

The objective of this study was to estimate the costeffectiveness of prophylaxis with posaconazole relative to fluconazole for the prevention of IFIs in allogeneic HSCT recipients receiving intensive immunosuppressive therapy for GVHD in the Netherlands. The analysis encompasses the payer perspective and was based on the RCT conducted by Ullmann and colleagues [11].

\section{Methods}

\section{Model structure}

A decision analytic model was developed (Fig. 1) to estimate the cost-effectiveness of orally administered posaconazole $(600 \mathrm{mg} /$ day $)$ versus standard fluconazole (400 mg/day) in patients with GVHD following HSCT treated with intensive immunosuppressive therapy. In line with the RCT by Ullmann et al. [11], the average age of patients was 40 years. Although the trial population consisted of $65 \%$ males, the model assumed an equal distribution of males and females because for the majority of source data used in the economic evaluation, only estimates for the total population (based on about $50 \%$ males and $50 \%$ females) were available. Consistent with the duration of the trial, clinical events during the first 112 days were modeled with a decision tree consisting of chance nodes reflecting the probability of a proven or probable IFI according to international consensus criteria, IFI related death, and death from other causes associated with posaconazole and fluconazole (see Fig. 1). The results of the 112-day trial were extrapolated to lifetime expected outcomes using a Markov state-transition structure consisting of repeated Markov cycles of 1 month. This allows for the estimation of the number of life years (LY) and quality adjusted life years (QALY) among patients that survived the prophylactic period. It was assumed that patients who survived an IFI during the prophylactic period displayed a monthly mortality rate similar to patients who did not experience an IFI.

With the model, the following outcomes were estimated for a patient treated with either posaconazole or fluconazole: (1) probability of an IFI; (2) life expectancy expressed in LYs; (3) expected QALYs, calculated as the LYs multiplied with the quality of life (or utility) of the of the underlying condition (i. e., GVHD); and (4) expected direct costs consisting of acquisition costs of antifungal drugs and costs of treating an IFI. In addition to these expected outcomes by treatment, differences in outcomes with posaconazole relative to fluconazole were estimated: (5) LYs gained, QALYs gained; (6) incremental costs; (7) and incremental costs expressed per LY or QALY gained (i.e., the incremental cost-effectiveness ratios (ICER)). Since the adverse event rate was similar for posaconazole and fluconazole in the RCT, costs and consequences due to adverse events were not included in this cost-effectiveness analysis.

\section{Source data}

Source data for the model analysis were obtained from the literature and validated in two separate consensus meetings with six Dutch clinical experts (one meeting with four and 


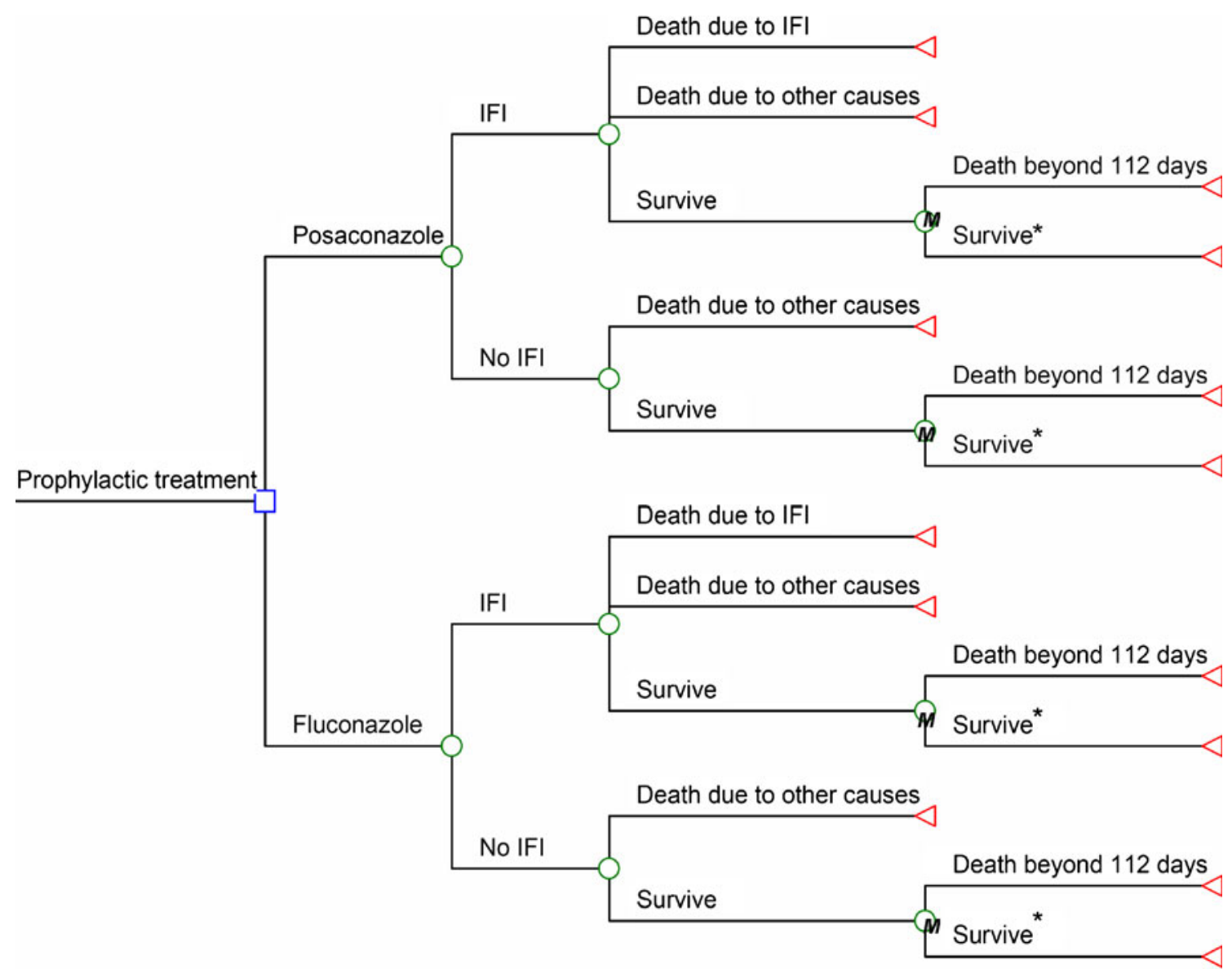

Fig. 1 Decision analytic model for cost-effectiveness evaluation of posaconazole versus fluconazole for prophylaxis of fungal infections. * survival beyond 112 days was extended with 1-month Markov cycles (M)

one meeting with two experts) including the co-authors J.J. W.M.J., E.L., and L.S.

The following data were used in the model (see Table 1):

1. The probability to contract an IFI during the 112-day period following initiation of prophylaxis with either posaconazole $(5.3 \%)$ or fluconazole $(9.0 \%)$ was obtained from Ullmann et al. [11].
2. Life years: the life expectancy per treatment arm was obtained by estimating the survival during as well as beyond the 112-day prophylactic period. The probability to survive the 112-day period following initiation of prophylaxis was estimated using data from Ullmann et al. [11]. The trial data provided the risk of dying due to an IFI as well as the probability of dying from other causes.
Table 1 Model input parameters for the base-case scenario along with the uncertainty distributions

Model input (mean and uncertainty range); for scenarios, see "Analysis" section

${ }^{\mathrm{a}}$ Mean values were obtained from the trial [11] and the low and high value was obtained by assuming a standard error of $10 \%$

\begin{tabular}{|c|c|c|c|c|}
\hline Parameter & Value & $\begin{array}{l}\text { Uncertainty } \\
\text { range }\end{array}$ & $\begin{array}{l}\text { Distribution used } \\
\text { for probabilistic } \\
\text { sensitivity analysis }\end{array}$ & Source \\
\hline Probability of IFI with fluconazole & 0.09 & $0.06 ; 0.12$ & Beta & {$[11]$} \\
\hline Probability of IFI with posaconazole & 0.05 & $0.03 ; 0.08$ & Beta & {$[11]$} \\
\hline Case-fatality of an IFI & 0.37 & $0.23 ; 0.52$ & Beta & {$[11]$} \\
\hline Probability of death from other causes & 0.25 & $0.21 ; 0.28$ & Beta & {$[11]$} \\
\hline Excess mortality after 112 days & 0.04 & - & - & {$[14]$} \\
\hline IFI treatment costs $€$ (non-responders) & $€ 37,204$ & $\begin{array}{r}€ 31,621 \\
€ 45,301\end{array}$ & Gamma & $\begin{array}{l}\text { [5] personal } \\
\text { communication }\end{array}$ \\
\hline Posaconazole treatment duration ${ }^{\mathrm{b}}$ & 80.3 & $64.6 ; 96.0$ & Gamma & {$[11]$} \\
\hline Fluconazole treatment duration $^{a}$ & 77.2 & $62.1 ; 92.3$ & Gamma & {$[11]$} \\
\hline $\begin{array}{l}\text { Utility (quality of life score for } \\
\text { underlying GVHD) }\end{array}$ & 0.9 & $0.75 ; 1.0$ & Uniform & {$[16]$} \\
\hline
\end{tabular}


The reported case fatality for IFI was significantly different between treatment arms $(0.25$ and 0.44 for posaconazole and fluconazole, respectively). However, since overall mortality was found to be similar between the two groups, we assumed equal case fatalities for the base-case analysis. Therefore, case-fatality rates were pooled for both treatment groups. The probability of death from other causes did not differ significantly between treatments either and were assumed to be equal for both treatment groups as well. The life expectancy beyond the 112-day period was assumed to follow that of chronic GVHD patients. Accordingly, a Weibull curve was fitted through the 10-year survival data of chronic GVHD patients as reported by Wingard and colleagues [14]. From this analysis, an annual excess mortality of $4 \%$ relative to the standard Dutch population was observed. Next, this annual excess mortality rate for GVHD was added to the age-specific death rates for the general Dutch population obtained from Dutch Life Tables [15] to estimate survival beyond the prophylactic period.

3. QALYs were obtained by multiplying the life expectancy in each treatment arm by the utility (or quality of life score) of the underlying condition. For chronic GVHD, a mean utility of 0.9 (range 0.75 to 1 ) was reported [16]. Nietert and colleagues estimated a similar value, for chronic GVHD [17]. Accordingly, it was assumed that if GVHD patients survived the 112 days following initiation of prophylaxis, the utility for chronic GVHD could be applied.

4. The costs incorporated in the model related to the costs of prophylaxis and treatment of an IFI. Doses of antifungal drugs used in the trial were similar to those used in daily clinical practice in the Netherlands. In the clinical trial, capsules of fluconazole were used. Since neither guidelines nor protocols suggest any preference of using a specific formulation of fluconazole, the base-case analysis was conducted with the less expensive formulation of capsules. In a separate scenario analysis, the cost of the oral suspension was used. To estimate the total cost of antifungal drug use, the average treatment duration with the antifungal drugs obtained from the trial was multiplied with the per-diem drug costs. The cost for posaconazole ( $600 \mathrm{mg}$, oral suspension) were $€ 92.86$ and for fluconazole (capsule) $€ 19.60$ [18]. An economic evaluation of the treatment of proven and probable invasive aspergillosis in the Netherlands by Jansen et al [5] reported expected costs for treatment with voriconazole, conventional amphotericin B, or itraconazole ( $€ 32,651, € 33,616$, and $€ 29,115$, respectively). These direct cost estimates were an average of responders and nonresponders to initial antifungal treatment and include costs associated with diagnosis, all antifungal drug treatment (first and switch treatments), monitoring, side effects, hospitalization, and outpatient care (for more details, see Jansen et al. [5]). For the type of patient considered in the current evaluation, these average cost estimates for an IFI by Jansen et al. are arguably an underestimation; the costs associated with treatment of IFIs among patients that received prophylactic treatment are probably better reflected by the cost estimates for nonresponders used in the study by Jansen et al. [5]. An estimate for this subgroup was obtained by contacting the authors of that study. After inflating this 2003 estimate to 2006, a cost of $€ 37,204$ was obtained which includes antifungal cost, hospitalization cost (including ICU stay), monitoring, and treatment of side effects. This estimate was applied to patients experiencing an IFI in the base-case scenario. In an alternative scenario, the average cost for responders and nonresponders as reported by Jansen et al. was used. All cost estimates were expressed in euros for the year 2006. In view of the fact that costs were incurred within the first year following the start of prophylaxis, discounting on costs was not applied. For outcomes, an annual discount rate of $1.5 \%$ for effects was applied in accordance with Dutch pharmacoeconomic guidelines [19].

\section{Analysis}

The source data are characterized by uncertainty. To incorporate this uncertainty in the evaluation, a probabilistic sensitivity analysis (PSA) was performed (Excel 2003/Visual basic) to quantify its effect on the model outcomes [20]. The uncertainty of the input parameters was reflected with a distribution that defined the probability of a certain value for the input parameters (Table 1). A random value from these input distributions was plugged in the model, and the corresponding expected costs and outcomes were calculated. This procedure was repeated 1,000 times to obtain uncertainty distributions of the expected outcomes. Each outcome was presented with a point estimate along with uncertainty reflected by the 2.5th and 97.5th percentile of the uncertainty distribution (the $95 \%$ uncertainty interval (UI)).

Posaconazole is considered cost-effective relative to fluconazole when the incremental cost per QALY gained is below a defined ceiling ratio of cost-effectiveness. For example, if the maximum willingness-to-pay (WTP) of a payer is $€ 50,000$ per QALY gained, then posaconazole is considered a cost-effective treatment when posaconazole is more efficacious and the ICER of posaconazole relative to fluconazole as estimated with the model is below this $€ 50,000$. Given the uncertainty in the source data and, therefore, the uncertainty in model estimates of cost-effectiveness, there is a certain probability that the incremental cost-effectiveness 
ratio is not below the WTP ceiling ratio. The lower the WTP ceiling ratio, the more difficult it is for a treatment to be considered cost-effective given its incremental costeffectiveness estimate. Acceptability curves were created to present the probability that posaconazole is cost-effective relative to fluconazole for different WTP ceiling ratios given the estimated ICERs and associated uncertainty.

Different scenarios were evaluated with the model. The base-case scenario was defined by applying the pooled IFI case fatality, IFI treatment costs of nonresponders, and fluconazole administered as a capsule. In addition to the base-case scenario, the following additional scenario analyses were performed:

1. No discounting of outcomes.

2. Fluconazole administered as oral suspension $(400 \mathrm{mg})$, $€ 27.89$ [18].

3. Instead of the conservatively assumed IFI case fatalities (equal for both treatments) treatment-specific IFIrelated deaths as observed in the trial was employed (i.e., $0.25(0.02-0.48)$ and $0.44(0.18-0.70)$ for posaconazole and fluconazole, respectively) [11].

4. Average IFI treatment costs as reported by Jansen et al. [5]: $€ 33,279$ ( $€ 29,211-37,347 ; 2,006$ euros).

5. Ullmann et al. noted that the observed incidence of IFIs in the trial was lower than those reported in other studies [11, 21, 22]. Consequently, in a scenario analysis, a risk of contracting an IFI of $15 \%$ was applied for fluconazole prophylaxis.

\section{Results}

Base-case scenario

Prophylaxis with posaconazole compared to fluconazole is expected to reduce the risk of IFIs with $4 \%$ (95\% UI $0-8 \%$;
Table 2). With an IFI case fatality of 0.37 and the life expectancy of the underlying condition, the observed reduction in IFIs resulted in an increase of 0.19 LYs $(95 \%$ UI 0.02-0.39) relative to fluconazole treatment. Taking into account the utility of the underlying condition, these differences translated into 0.17 QALYs (95\% UI 0.02 0.36 ) gained with posaconazole (Table 2).

The expected direct medical costs per patient were greater with posaconazole than with fluconazole prophylaxis. The costs with posaconazole were primarily incurred during the prophylactic period (79\%), whereas the majority of costs with fluconazole related to treatment of IFIs (69\%) due to unsuccessful prophylaxis (Table 2). The ICER of posaconazole versus fluconazole is estimated to be $€$ 24,016 per LY gained and $€ 26,225$ per QALY gained. (€4,566; 95\% UI $€ 2,460-6,854)$

In Fig. 2, the probability that posaconazole is cost-effective relative to fluconazole is presented for different WTP ratios. Considering a WTP threshold of $€ 50,000$ per QALY, there is a $79 \%$ probability that posaconazole is cost-effective in comparison to fluconazole.

\section{Alternative scenarios}

The results of the alternative scenario analyses are presented in Table 3. When discounting was discarded, the incremental QALYs and LYs slightly increased. Applying treatment-specific case fatalities for IFIs, as reported by Ullmann et al. [11], almost doubled the incremental LYs and QALYs gained with posaconazole. The incremental cost-effectiveness ratio of posaconazole versus fluconazole decreased to $€ 13,519$ per LY gained and $€ 14,868$ per QALY gained. The scenario analysis assuming a higher IFI risk $(15 \%)$ resulted in a reduction in incremental costs of posaconazole versus fluconazole and also increased incremental LYs and QALYs. For this scenario, the incremental cost-effectiveness ratio of pos-
Table 2 Expected outcomes and costs per patient

\footnotetext{
a The uncertainty range is based on the 2.5th and 97.5th percentile of the uncertainty distribution created with a probabilistic sensitivity analysis of 1,000 simulations
}

\begin{tabular}{llll}
\hline & $\begin{array}{l}\text { Posaconazole mean } \\
(95 \% \text { UI })\end{array}$ & $\begin{array}{l}\text { Fluconazole mean } \\
(95 \% \text { UI })\end{array}$ & $\begin{array}{l}\text { Difference mean } \\
(95 \% \text { UI })^{\mathrm{a}}\end{array}$ \\
\hline Probability of an IFI & 0.05 & 0.09 & -0.04 \\
Life expectancy in years & $(0.03 ; 0.08)$ & $(0.06 ; 0.12)$ & $(-0.08 ; 0.00)$ \\
Quality Adjusted Life Years & 11.66 & 11.47 & 0.19 \\
& $(11.10 ; 12.19)$ & $(10.88 ; 12.02)$ & $(0.02 ; 0.39)$ \\
Total treatment cost & 10.52 & 10.35 & 0.17 \\
& $€ 9.83 ; 11.74)$ & $(8.67 ; 11.60)$ & $(0.02 ; 0.36)$ \\
\% Prophylactic drug costs & $(7,743 ; 11,388)$ & $€ 4,861$ & $(2,460 ; 6,854)$ \\
\% IFI treatment costs & $79 \%(€ 7,457)$ & $31 \%(€ 1,513)$ & \\
\hline
\end{tabular}


Fig. 2 Acceptability curves for the base case and the $15 \%$ IFI background risk scenarios, representing the probability that posaconazole is cost-effective in comparison to fluconazole for different values of willingness to pay for a QALY

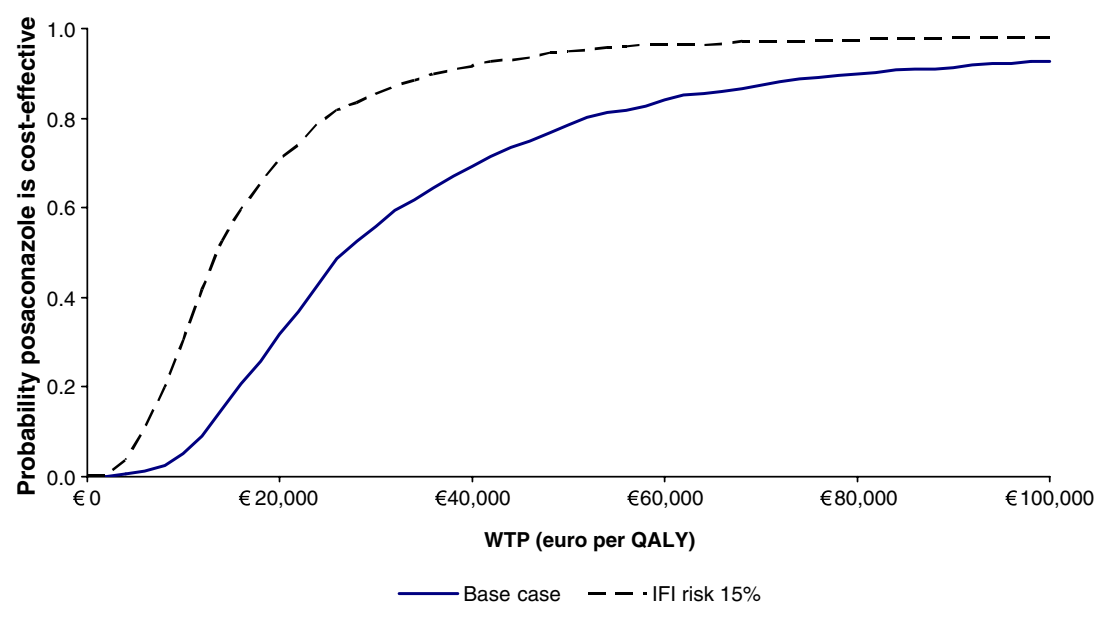

aconazole versus fluconazole decreased to $€ 12,255$ per LY gained and $€ 13,462$ per QALY gained and the corresponding probability of cost-effectiveness at a WTP of $€ 20,000$ and $€ 50,000$ per QALY increased to $71 \%$ and $95 \%$, respectively (Fig. 2).

When average first-line IFI treatment costs as reported by Jansen et al. [5] were applied, the incremental costs of posaconazole versus fluconazole slightly increased; however, the probability of cost-effectiveness remained similar.

\section{Discussion}

Cost-effectiveness analysis aims to provide information on the expected costs and outcomes of a new intervention relative to the accepted or standard intervention. When it is said that an intervention is considered cost-effective, it does not necessarily mean that the new treatment is cost-saving relative to the standard intervention. The total costs associated with the new treatment can be higher than with the standard intervention and still be considered costeffective. This is the case if the new treatment significantly enhances quality of life and/or life expectancy over and above the current standard. The current cost-effectiveness evaluation applies to the Dutch situation of prophylaxis of invasive fungal infections among patients with GVHD. Prophylaxis with posaconazole compared to fluconazole resulted in a gain of 0.19 LYs and 0.17 QALYs corresponding to $€ 24,016$ per LY gained and $€ 26,225$ per QALY gained, respectively. This ICER reflects that posaconazole can be considered cost-effective relative to fluconazole in the Netherlands.

Analysis of alternative scenarios, such as fluconazole as oral suspension, no discounting, and applying the first-line IFI treatment costs as reported by Jansen et al. [5], resulted in similar or improved cost-effectiveness estimates of posaconazole relative to fluconazole. De Pauw et al. commented that centers with a high incidence of invasive aspergillosis are more likely to adopt antifungal prophylaxis as a strategy [23]. In the trial of Ullmann et al., the authors noted that the risk of contracting an IFI in their study was lower than that reported in other studies, which could be due to the fact that all patients in the Ullmann trial received prophylaxis, and not all studies used the consensus criteria; some studies included possible cases as well [11]. Consequently, the background risk of contracting an IFI in the clinical setting may be higher than the risk as reported by Ullmann and used in the base-case analysis. Applying a higher background risk of contracting an IFI (15\%) reduced the ICER of posaconazole to $€ 13.462$ per QALY, a more favorable result.

The study was performed from the payer perspective, which in this case is comparable to the societal perspective. Since the patients considered were not employed due to the severity of the underlying disease, indirect costs were not included. Furthermore, costs for the underlying disease were not included because the interest was in the additional costs of prophylaxis and avoidance of IFIs.

In order to develop an economic model for antifungal prophylaxis, several simplifications and assumptions were made with respect to routine practice. An important assumption is the application of survival as well as utility estimates for chronic GVHD for patients that survived the 112-day prophylactic period. Although acute GVHD is an important risk factor for chronic GVHD [24, 25], it is unlikely that all of the acute GVHD patients from the clinical trial by Ullmann et al. develop chronic GVHD [11]. Consequently, the life expectancy as well as the quality-oflife of patients surviving the prophylactic period will be underestimated and the cost-effectiveness as well. Furthermore, the Wingard data on survival originated from 1989; the impact of any developments that improved care and possibly survival since then were not taken into account [14]. For the base-case scenario, it was assumed that the prophylactic regimen did not affect the case fatality of an IFI. Since the RCT conducted by Ullmann et al. reported a 
higher IFI case fatality for fluconazole than for posaconazole, the assumption of equal case fatality will bias against posaconazole. Indeed, when treatment-specific IFI case fatalities were applied, the cost per QALY gained almost halved $(€ 14,868)$.

For IFIs that occur despite prophylaxis, the current model assumes similar IFI treatment costs for each treatment arm. This assumption might result in a bias favoring posaconazole prophylaxis, since "failures" on a broad spectrum prophylactic agent, like posaconazole, might be considered more complicated than those failing on fluconazole prophylaxis. Consequently, patients who fail on posaconazole might require treatment with more expensive antifungal agents. However, since posaconazole prophylaxis is not established, routine, practice data cannot confirm this (expert opinion); therefore, similar IFI treatment costs were assumed to reflect current clinical practice.

Efficacy of posaconazole versus fluconazole was obtained from a RCT [11]. Although such a design provides high quality of evidence, the actual estimates are still characterized by (sampling) uncertainty (as represented by $95 \%$ confidence intervals). Utility and resource use data used in the evaluation were extracted from other studies than the Ullmann trial, adding additional uncertainty. Model-based economic evaluations only provide value if the uncertainties in the combination of different sources of data are made explicit, and their impact is investigated. Consequently, a PSA was conducted to quantify the uncertainty in the expected costs, outcomes, and costeffectiveness based on the uncertainty in the source data. For the base-case analysis, the probability of posaconazole being cost-effective compared to fluconazole was only $32 \%$ at a conservative WTP threshold of $€ 20,000$ per QALY. However, in many countries including the Netherlands, the WTP thresholds are not absolute limits, since the severity of the disease is also a criterion that is considered [26-28]. Therefore, the WTP threshold for antifungal prophylaxis can be expected to be considerably higher than $€ 20,000$ per QALY. Using a WTP threshold of $€ 50,000$ or even $€ 75,000$ per QALY resulted in a higher probability of $79 \%$ and $89 \%$, respectively, that posaconazole prophylaxis is cost-effective relative to fluconazole. A higher background risk for IFIs of $15 \%$ increased the probability of posaconazole being a costeffective option: a probability of $95 \%$ at a WTP level of $€ 50,000$ per QALY was observed. Furthermore, IFIs can be considered to pose a serious risk to the investments related to HSCT. The estimated costs of $€ 151,754$ (1998 price level) per patient for the first 2 years after transplantation, should be considered when deciding on any WTP ratio regarding cost-effectiveness for prophylaxis [29]. Although the current analysis considered the official list prices of antifungal drugs, actual acquisition cost of the drugs in a hospital may of course differ. However, additional analysis 
demonstrated that differences in drug acquisition costs only had a minor impact on the cost effectiveness (data not shown).

Considering the medical necessity as well as the investments made for allogeneic HSCT, posaconazole can be considered cost-effective relative to fluconazole for prophylaxis of IFI in patients with GVHD in the Netherlands. The cost-effectiveness of prophylactic posaconazole depends on the IFI risk, which can vary by hospital.

Acknowledgements The authors would like to thank B. Rijnders (Internal Medicine, Section of Infectious Diseases, Erasmus MC, Rotterdam), Ad W. Dekker (Hematology, UMC Utrecht), and Arnold G. Vulto (Pharmacy, Erasmus MC Rotterdam) for their valuable contribution during the expert meetings on the economic evaluation. This study was funded by Schering Plough.

Open Access This article is distributed under the terms of the Creative Commons Attribution Noncommercial License which permits any noncommercial use, distribution, and reproduction in any medium, provided the original author(s) and source are credited.

\section{References}

1. Richardson MD (2005) Changing patterns and trends in systemic fungal infections. J Antimicrob Chemother 56(Suppl 1):i5-i11

2. Hagen EA, Stern H, Porter D, Duffy K, Foley K, Luger S, Schuster SJ, Stadtmauer EA, Schuster MG (2003) High rate of invasive fungal infections following nonmyeloablative allogeneic transplantation. Clin Infect Dis 36:9-15

3. Torres HA, Hachem RY, Chemaly RF, Kontoyiannis DP, Raad II (2005) Posaconazole: a broad-spectrum triazole antifungal. Lancet Infect Dis 5:775-785

4. Caggiano V, Weiss RV, Rickert TS, Linde-Zwirble WT (2005) Incidence, cost, and mortality of neutropenia hospitalization associated with chemotherapy. Cancer 103:1916-1924

5. Jansen JP, Meis JF, Blijlevens NM, van't Wout JW (2005) Economic evaluation of voriconazole in the treatment of invasive aspergillosis in the Netherlands. Curr Med Res Opin 21:1535-1546

6. Goodman JL, Winston DJ, Greenfield RA, Chandrasekar PH, Fox B, Kaizer H, Shadduck RK, Shea TC, Stiff P, Friedman DJ (1992) A controlled trial of fluconazole to prevent fungal infections in patients undergoing bone marrow transplantation. N Engl J Med 326:845-851

7. Slavin MA, Osborne B, Adams R, Levenstein MJ, Schoch HG, Feldman AR, Meyers JD, Bowden RA (1995) Efficacy and safety of fluconazole prophylaxis for fungal infections after marrow transplantation-a prospective, randomized, double-blind study. J Infect Dis 171:1545-1552

8. Dixon DM, McNeil MM, Cohen ML, Gellin BG, La Montagne JR (1996) Fungal infections: a growing threat. Public Health Rep 111:226-235

9. Edmond MB, Wallace SE, McClish DK, Pfaller MA, Jones RN, Wenzel RP (1999) Nosocomial bloodstream infections in United States hospitals: a three-year analysis. Clin Infect Dis 29:239-244

10. Groll AH, Walsh TJ (2001) Uncommon opportunistic fungi: new nosocomial threats. Clin Microbiol Infect 7(Suppl 2):8-24

11. Ullmann AJ, Lipton JH, Vesole DH, Chandrasekar P, Langston A, Tarantolo SR, Greinix H, Morais dA, Reddy V, Boparai N, Pedicone L, Patino H, Durrant S (2007) Posaconazole or fluconazole for prophylaxis in severe graft-versus-host disease. N Engl J Med 356:335-347

12. Cornely OA, Maertens J, Winston DJ, Perfect J, Ullmann AJ, Walsh TJ, Helfgott D, Holowiecki J, Stockelberg D, Goh YT, Petrini M, Hardalo C, Suresh R, Angulo-Gonzalez D (2007) Posaconazole vs. fluconazole or itraconazole prophylaxis in patients with neutropenia. N Engl J Med 356:348-359

13. Stam WB, O'Sullivan AK, Rijnders B, Lugtenburg E, Span LF, Janssen JJ, Jansen JP (2008) Economic evaluation of posaconazole versus standard azole prophylaxis in high risk neutropenic patients in the Netherlands. Eur J Haematol 81(6):467-474

14. Wingard JR, Piantadosi S, Vogelsang GB, Farmer ER, Jabs DA, Levin LS, Beschorner WE, Cahill RA, Miller DF, Harrison D (1989) Predictors of death from chronic graft-versus-host disease after bone marrow transplantation. Blood 74:1428-1435

15. Centraal Bureau voor de Statistiek. CBS STATLINE Overlevingstafels 2005. http://statline.cbs.nl/StatWeb/publication/?DM=SLNL $\& P A=37360$ ned $\& D 1=3 \& D 2=a \& D 3=a \& D 4=55 \& H D R=G 1$, $\mathrm{T} \& \mathrm{STB}=\mathrm{G} 3, \mathrm{G} 2 \& \mathrm{VW}=\mathrm{T}$

16. Lee SJ, Kuntz KM, Horowitz MM, McGlave PB, Goldman JM, Sobocinski KA, Hegland J, Kollman C, Parsons SK, Weinstein MC, Weeks JC, Antin JH (1997) Unrelated donor bone marrow transplantation for chronic myelogenous leukemia: a decision analysis. Ann Intern Med 127:1080-1088

17. Nietert PJ, Silverstein MD, Abboud MR (2002) Sickle cell anaemia: epidemiology and cost of illness. Pharmacoeconomics 20:357-366

18. Z-index B.V. (2007) Z-Index taxe: selection of the G standard drug databank. Z-index B.V., The Haag

19. College voor Zorgverzekeringen (2006) Richtlijnen voor farmacoeconomisch onderzoek, geactualiseerde versie. College voor Zorgverzekeringen, Diemen

20. Briggs A, Sculpher M, Buxton M (1994) Uncertainty in the economic evaluation of health care technologies: the role of sensitivity analysis. Health Econ 3:95-104

21. Jantunen E, Ruutu P, Niskanen L, Volin L, Parkkali T, KoukilaKahkola P, Ruutu T (1997) Incidence and risk factors for invasive fungal infections in allogeneic BMT recipients. Bone Marrow Transplant 19:801-808

22. Marr KA, Carter RA, Crippa F, Wald A, Corey L (2002) Epidemiology and outcome of mould infections in hematopoietic stem cell transplant recipients. Clin Infect Dis 34:909-917

23. De Pauw BE, Donnelly JP (2007) Prophylaxis and aspergillosis-has the principle been proven? N Engl J Med 356:409-411

24. Carlens S, Ringden O, Remberger M, Lonnqvist B, Hagglund H, Klaesson S, Mattsson J, Svahn BM, Winiarski J, Ljungman P, Aschan J (1998) Risk factors for chronic graft-versus-host disease after bone marrow transplantation: a retrospective single centre analysis. Bone Marrow Transplant 22:755-761

25. Remberger M, Kumlien G, Aschan J, Barkholt L, Hentschke P, Ljungman P, Mattsson J, Svennilson J, Ringden O (2002) Risk factors for moderate-to-severe chronic graft-versus-host disease after allogeneic hematopoietic stem cell transplantation. Biol Blood Marrow Transplant 8:674-682

26. Jonsson B (2004) Changing health environment: the challenge to demonstrate cost-effectiveness of new compounds. Pharmacoeconomics 22(Suppl 4):5-10

27. Council for Public Health and Healthcare (2006) Sensible and sustainable care. Council for Public Health and Healthcare, The Hague

28. Stolk EA, Poley MJ (2005) Criteria for determining a basic health services package. Recent developments in The Netherlands. Eur J Health Econ 6:2-7

29. Groot MT, van Agthoven M, Lowenberg B, Willemze R (2004) Uylde Groot CA [The role of cost analysis in the evaluation of the development of medical technology. The case of allogenic stem-cell transplantation]. Ned Tijdschr Geneeskd 148:480-484 